\title{
Image Retrieval Based On Color and Texture Features of the Image Sub-blocks
}

\author{
Ch.Kavitha \\ Associate Professor \\ IT Department \\ Gudlavalleru Engineering \\ College, Gudlavalleru, A.P, \\ India
}

\author{
Dr.B.Prabhakara Rao \\ Professor\&Director of \\ Evaluation \\ JNTUK, Kakinada, A.P, India
}

\author{
Dr.A.Govardhan \\ Professor\&Principal \\ JNTUH college of Engineering \\ Jagtial, A.P, India
}

\begin{abstract}
Nowadays people are interested in using digital images. So the size of the image database is increasing enormously. Lot of interest is paid to find images in the database. There is a great need for developing an efficient technique for finding the images. In order to find an image, image has to be represented with certain features. Color and texture are two important visual features of an image. So, an efficient image retrieval technique which uses local color and texture features is proposed. An image is partitioned into sub-blocks of equal size as a first step. Color of each sub-block is extracted by quantifying the HSV color space into non-equal intervals and the color feature is represented by cumulative histogram. Texture of each sub-block is obtained by using gray level cooccurrence matrix. A one to one matching scheme is used to compare the query and target image. Euclidean distance is used in retrieving the similar images. The efficiency of the method is demonstrated with the results.
\end{abstract}

\section{General Terms}

Algorithm, search, match.

\section{Keywords}

Image retrieval, color, texture, cumulative histogram, gray level co-occurrence matrix.

\section{INTRODUCTION}

Content-based image retrieval (CBIR)[1] has become a prominent research topic because of the proliferation of video and image data in digital form. Increased bandwidth availability to access the internet in the near future will allow the users to search for and browse through video and image databases located at remote sites. Therefore fast retrieval of images from large databases is an important problem that needs to be addressed.

Image retrieval systems attempt to search through a database to find images that are perceptually similar to a query image. CBIR is an important alternative and complement to traditional text-based image searching and can greatly enhance the accuracy of the information being returned. It aims to develop an efficient visual-Content-based technique to search, browse and retrieve relevant images from large-scale digital image collections. Most proposed CBIR techniques automatically extract low-level features (e.g. color, texture, shapes and layout of objects) to measure the similarities among images by comparing the feature differences.

The CBIR system based on HSV color histogram and GLCM texture. But there is a focus on global features. From the literature we came to know that the local features $[4,5,6]$ play an important role in finding the similarity of images than global features $[2,3,4]$. So, in this paper, we present a technique for image retrieval based on local color and texture. Because Low level visual features of the images such as color and texture are especially useful to represent and to compare images automatically. In the concrete selection of color and texture description, we use color histogram, Gray-level cooccurrence matrix.

Texture is also an important visual feature that refers to innate surface properties of an object and their relationship to the surrounding environment. Many objects in an image can be distinguished solely by their textures without any other information. There is no universal definition of texture. Texture may consist of some basic primitives, and may also describe the structural arrangement of a region and the relationship of the surrounding regions [17]. In our approach we have used the statistic texture features using gray-level cooccurrence matrix (GLCM).

So a technique is developed which captures color and texture features of sub-blocks of the image. For each subblock cumulative histogram and static texture features using GLCM are determined. A one to one integrated matching procedure is used to find the image similarity.

The section II outlines proposed method. The section III deals with experimental setup. The section IV presents results. The section V presents conclusions.

\section{PROPOSED METHOD}

The proposed method is based on color and texture features of image sub-blocks with one to one matching. The algorithm for the proposed method is given below:

The algorithm for the proposed method:

Step1: divide each image in the database and the target image into 6-equal sized sub-blocks.

Step2: for each sub-block construct cumulative HSV color histogram.

Step3: for each sub-block obtain four statistic features (Energy, Contrast, Entropy and inverse difference) from GLCM.

Step4: construct a combined feature vector for color and texture.

Step5: find the distances between feature vector of query 
image and the feature vectors of target images using normalized Euclidean distance.

Step6: sort the Euclidean distances.

Step7: retrieve first 20 most similar images with minimum distance

\section{1 partitioning the image into sub-blocks}

Firstly the image is partitioned into $6(2 \times 3)$ equal sized sub-blocks as shown in Fig.1. The size of the subblock in an image of size $256 \times 384$ is $128 \times 128$. The images with other than $256 \times 384$ sizes are resized to $256 \times 384$.

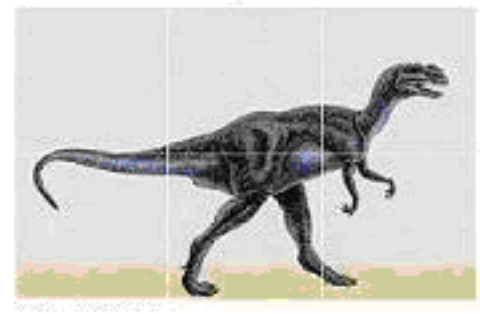

128X128 sub-blocks

\section{6 sub-blocks Color and Texture features}

Fig. 1 partitioned image

\subsection{Extraction of color of an image sub-block}

Color is an important visual attribute for both human perception and computer vision and one of the most widely used visual features in image/video retrieval [18]. An appropriate color space and quantization must be specified along with the histogram representation. In the HSV color space [9], hue is used to distinguish colors, saturation is the percentage of white light added to a pure color and value refers to the perceived light intensity [10]. Therefore we have opted the HSV color space to extract the color features according to hue, saturation and value. We observed that HSV color space is better than the RGB color model for our approach using the following quantization scheme where each component is quantized with non-equal intervals: $\mathrm{H}$ : 8 bins; $\mathrm{S}: 3$ bins and $\mathrm{V}: 3$ bins. Finally we concatenate $8 \times 3 \times 3$ histogram and get 72-dimensional vector [16]. To reduce the number of zeros in the histogram, we adopted the cumulative histogram. The procedure is as follows:

In the HSV color space, each component occupies a large range of values. If we use direct values of $\mathrm{H}, \mathrm{S}$ and $\mathrm{V}$ components to represent the color feature, it requires lot of computation. So it is better to quantify the HSV color space into non-equal intervals. At the same time, because the power of human eye to distinguish colors is limited, we do not need to calculate all segments. Unequal interval quantization according the human color perception has been applied on $H$, $S$, and $V$ components.

Based on the color model of substantial analysis, we divided Hue into eight parts. Saturation and intensity is divided into three parts separately in accordance with the human eyes to distinguish [9, 12, and 13]. In accordance with the different colors and subjective color perception quantification, quantified hue $(\mathrm{H})$, saturation $(\mathrm{S})$ and value (V) are showed as equation 1 .

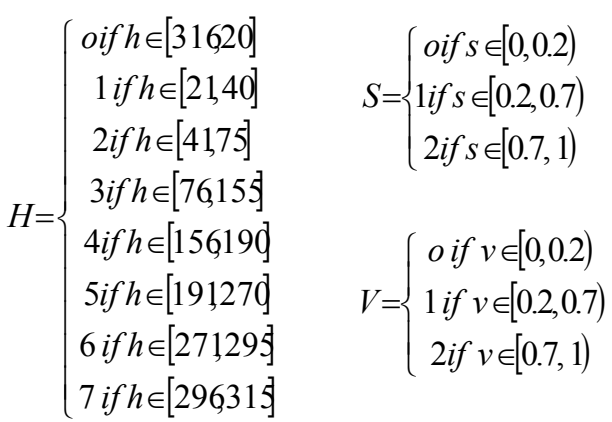

In accordance with the quantization level above, threedimensional feature vector for different values of $\mathrm{H}, \mathrm{S}, \mathrm{V}$ with different weight to form one-dimensional feature vector named G:

$$
G=Q s Q v H+Q v S+V
$$

Where $Q_{s}$ is quantified series of $\mathrm{S}, Q_{v}$ is quantified series of V. Here we set $Q_{s}=Q_{v}=3$, then

$$
G=9 H+3 S+V
$$

In this way, three-component vector of HSV form Onedimensional vector, which quantize the whole color space for the 72 kinds of main colors. So we can handle 72 bins of onedimensional histogram. This quantification can be effective by reducing the computational time and complexity. It will be much of the deviation of the calculation of the similarity if we do not normalize, so we must normalize the components to the same range. The process of normalization is to make the components of feature vector equal importance.

Color histogram is derived by first quantizing colors in the image into 72 bins in HSV color space. Then counting the number of image pixels in each bin. One of the weaknesses of color histogram is that when the characteristics of images should not take over all the values, the statistical histogram will appear in a number of zero values. The emergence of these zero values would make similarity measure does not accurately reflect the color difference between images and statistical histogram method to quantify more sensitive parameters. Therefore, this paper represents the onedimensional vector $\mathrm{G}$ by constructing a cumulative histogram of the color characteristics of image after using non-interval HSV quantization $[12,13]$ for $\mathrm{G}$.

\subsection{Extraction of texture of an image sub-block}

GLCM $[11,13]$ is created in four directions with the distance between pixels as one. Texture features are extracted from the statistics of this matrix. Four GLCM texture features are commonly used which are given below:

GLCM is composed of the probability value, it is defined by which expresses $P(i, j \mid d, \theta)$ the probability of the couple pixels at $\theta$ direction and $d$ interval. When $\theta$ and $d$ is determined, is showed by $P_{i, j}$. Distinctly GLCM is a symmetry matrix and its level is determined by the image gray-level. Elements in the matrix are computed by the equation shown below: 


$$
P(i, j \mid d, \theta)=\frac{P(i, j \mid d, \theta)}{\sum_{i} \sum_{j} P(i, j \mid d, \theta)}
$$

GLCM expresses the texture feature according the correlation of the couple pixels gray-level value at different positions. It quantificationally describes the texture feature. In this paper, four texture features are considered. They include energy, contrast, entropy, inverse difference.

$$
\text { Energy } E=\sum_{x} \sum_{y} P(x, y)^{2}
$$

It is a texture measure of gray-scale image represents homogeneity changing, reflecting the distribution of image gray-scale uniformity of weight and texture.

$$
\text { Contrast } I=\sum \sum(x-y)^{2} P(x, y)
$$

Contrast is the main diagonal near the moment of inertia, which measures how the values of the matrix are distributed and number of images of local changes reflecting the image clarity and texture of shadow depth. Large Contrast represents deeper texture.

$$
\text { Entropy } S=-\sum_{x} \sum_{y} P(x, y) \log P(x, y)
$$

Entropy measures randomness in the image texture. Entropy is minimum when the co-occurrence matrix for all values is equal. On the other hand, if the value of co-occurrence matrix is very uneven, its value is greater. Therefore, the maximum entropy implied by the image gray distribution is random.

Inverse difference $H=\sum_{x} \sum_{y} \frac{1}{1+(x-y)^{2}} P(x, y)$

It measures number of local changes in image texture. Its value in large is illustrated that image texture between the different regions of the lack of change and partial very evenly. Here $p(x, y)$ is the gray-level value at the Coordinate $(x, y)$.

Four texture features are computed for all the tiles of an image and is used as feature descriptor. Combined feature vector of Color and texture is formulated for each sub-block.

\subsection{Integrated image matching}

We have used an integrated image matching procedure (i.e. one to one matching) similar to the one used in [7, 14]. The image is divided into sub-blocks.

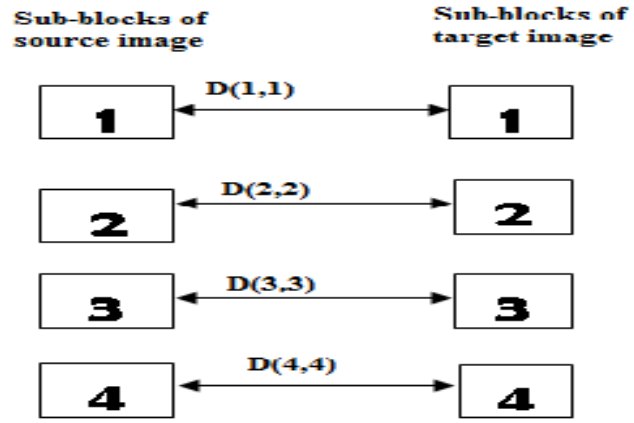

Fig. 2: graph showing 4 sub-blocks of target and query images.
With the decomposition of the image, the number of subblocks remains same for all the images. The sub-blocked approach proposed here is similar to the one used in [8]. The matching is done by comparing sub-blocks of query image with the sub-blocks of target image in corresponding positions. The graph is formulated like Fig.2 to depict the distances between sub-blocks of query image and target images.

\section{EXPERIMENTAL SETUP}

3.1 Data set: Wang's [15] dataset comprising of 1000 Corel images with ground truth. The image set comprises 100 images in each of 10 categories. The images are of the size $256 \times 384$ or 384 X256. But the images with $384 X 256$ are resized to $256 \times 384$.

3.2 Feature set: The feature set comprises color and texture descriptors computed for each sub-block of an image as we discussed in section 2 .

\subsection{Computation of similarity}

The similarity between sub-blocks of query and target image is measured from two types of characteristic features which includes color and texture features to formulate the graph. Matching of the sub-blocks is done based on one to one matching. Two types of characteristics of images represent different aspects of property. So during the Euclidean similarity measure, when necessary the appropriate weights to combine them are also considered.

Therefore, in carrying out Euclidean similarity measure we should consider necessary appropriate weights to combine them. We construct the Euclidean calculation model as follows:

$$
D(A, B)=\omega_{1} D\left(F_{C A}, F_{C B}\right)+\omega_{2} \mathrm{D}\left(F_{T A}, F_{T B}\right)
$$

Here $\omega_{1}$ is the weight of color features, $\omega_{2}$ is the weight of texture features, $F_{C A}$ and $F_{C B}$ represents the normalized 72dimensional color features for image A and B. For a method based on GLCM, $F_{T A}$ and $F_{T B}$ on behalf of 4- dimensional normalized texture features correspond to image $\mathrm{A}$ and $\mathrm{B}$. Here, we combine color features and texture features. The value of $\omega$ through experiments shows that at the time $\omega_{1}=\omega_{2}=0.5$ has better retrieval performance.

\section{EXPERIMENTAL RESULTS}

The experiments were carried out as explained in sections 2 and 3 .

The results are benchmarked with some of the existing systems using the same database[15]. The quantitative measure is given below

$$
p(i)=\frac{1}{100} \sum_{1 \leq j \leq 1000, r(i, j) \leq 100, I D(j)=I D(i)} 1
$$

Where $p(i)$ is precision of query image $\operatorname{I}, \operatorname{ID}(\mathrm{i})$ and $\operatorname{ID}(\mathrm{j})$ are category ID of image I and $\mathrm{j}$ respectively, which are in the range of 1 to 10 . the $r(i, j)$ is the rank of image $j$. This value is percentile of images belonging to the category of image $i$, in the forst 100 retrieved images.

The average precision $p_{t}$ for category $\boldsymbol{t}(1 \leq \mathrm{t} \leq 10)$ is given by 


$$
p_{t}=\frac{1}{100} \sum_{1 \leq i \leq 1000, I D(i)=t} p(i)
$$

The comparison of proposed method with other retrieval systems is presented in the Table 1 . These retrieval systems are based on HSV color, GLCM texture and combined HSV color and GLCM texture. Our sub-blocks based retrieval system is better than these systems in all categories of the database.

The experiments were carried out on a Core i3, $2.4 \mathrm{GHz}$ processor with 4GB RAM using MATLAB.

Only simple features of image information can not get comprehensive description of image content. We consider the color and texture features combining not only be able to express more image information, but also to describe image from the different aspects for more detailed information in order to obtain better search results. Retrieval algorithm flow is as follows:

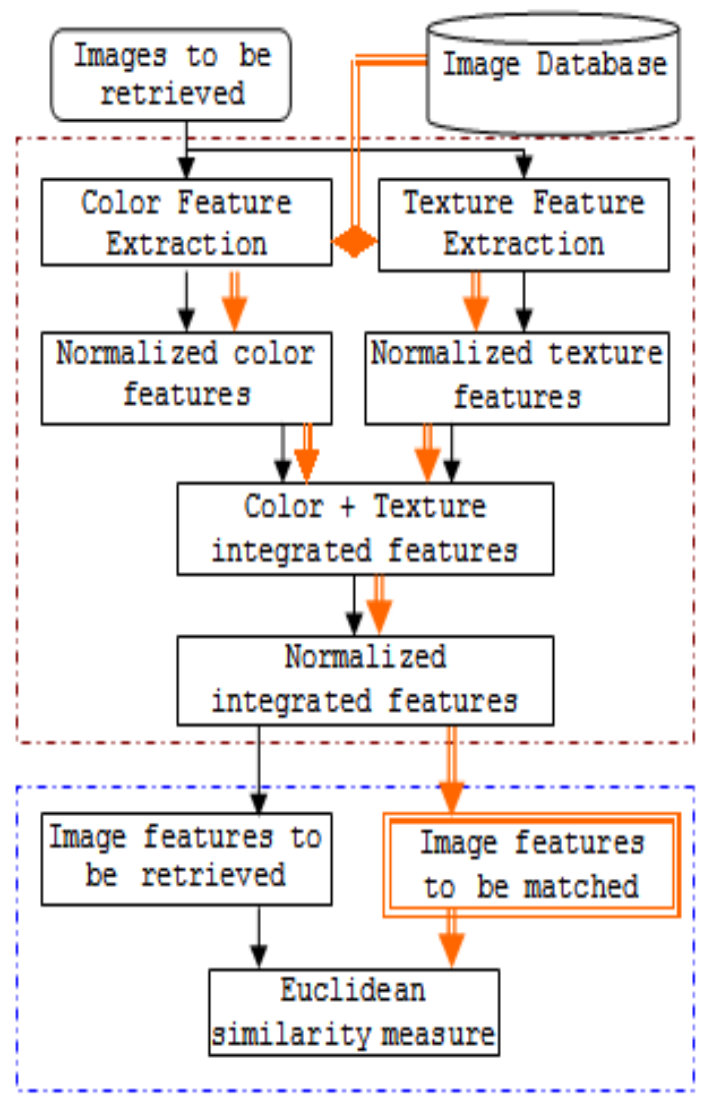

Fig 3. Algorithm scheme

Table1. Comparison of average precision obtained by proposed method with other retrieval systems. In our data base, there are ten categories. In each class of images, one can observe the improvement in the average precision value by our proposed method when compared with other techniques.
The graph in Fig.4 shows the Comparison of average precision obtained by proposed method with other retrieval systems.

Table1. Average precision

\begin{tabular}{|l|l|l|l|l|}
\hline \multirow{2}{*}{ Class } & \multicolumn{5}{|c|}{ Average Precision } \\
\cline { 2 - 5 } & $\begin{array}{l}\text { HSV } \\
\text { color }\end{array}$ & $\begin{array}{l}\text { GLCM } \\
\text { Texture }\end{array}$ & $\begin{array}{l}\text { HSV } \\
\text { color } \\
\text { +GLCM } \\
\text { Texture }\end{array}$ & $\begin{array}{l}\text { HSVcolor } \\
\text { +GLCM Texture } \\
\text { of image sub- } \\
\text { blocks(proposed } \\
\text { method) }\end{array}$ \\
\hline Africa & 0.36 & 0.21 & 0.34 & 0.41 \\
\hline Beaches & 0.27 & 0.35 & 0.21 & 0.32 \\
\hline Building & 0.38 & 0.50 & 0.24 & 0.37 \\
\hline Bus & 0.45 & 0.22 & 0.51 & 0.66 \\
\hline Dinosaur & 0.26 & 0.29 & 0.39 & 0.43 \\
\hline Elephant & 0.3 & 0.24 & 0.26 & 0.39 \\
\hline Flower & 0.65 & 0.73 & 0.81 & 0.87 \\
\hline Horses & 0.19 & 0.25 & 0.28 & 0.35 \\
\hline Mountain & 0.15 & 0.18 & 0.20 & 0.34 \\
\hline Food & 0.24 & 0.29 & 0.25 & 0.31 \\
\hline
\end{tabular}

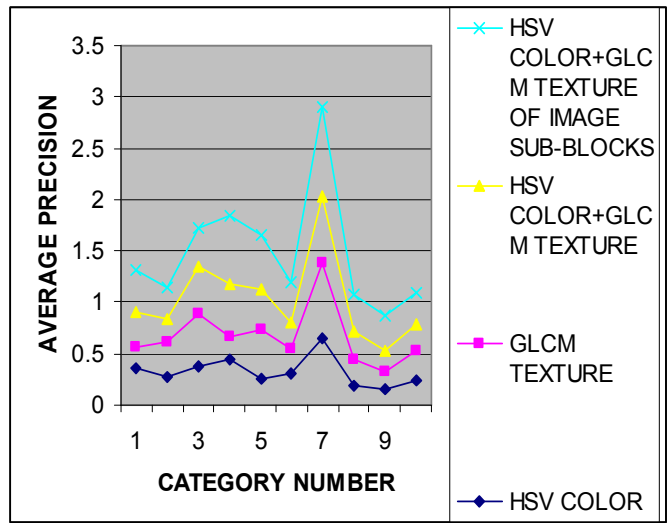

Fig. 4 Average precision of various image retrieval methods.

\section{CONCLUSIONS}

In this paper a new image retrieval method based on HSV color and GLCM texture features of image sub-blocks with one to one matching is proposed. We combined color and texture features with normalized Euclidean distance. Our experimental results demonstrate that the proposed method based on color and texture features of image sub-blocks has better retrieval performance compared with the Image retrieval system using only HSV color, only GLCM texture and combined HSV color and GLCM texture. As further studies, the proposed retrieval method is to be evaluated with other integrated matching techniques. 


\section{REFERENCES}

[1]. Ritendra Datta, Dhiraj Joshi, Jia Li and James Wang, "Image Retrieval:Ideas, Influences and trends of the New Age", Proceedings of the $7^{\text {th }}$ ACM SIGMM international workshop on multimedia information retrieval, Novenber 10-11,2005, Hilton, Singapore

[2] W. Niblack et al., "The QBIC Project: Querying Images by Content Using Color, Texture, and Shape," in Proc. SPIE, vol. 1908, San Jose, CA, pp. 173-187, Feb. 1993.

[3] A. Pentland, R. Picard, and S. Sclaroff, "Photobook: Content-based Manipulation of Image Databases," in Proc. SPIE Storage and Retrieval for Image and Video Databases II, San Jose, CA, pp. 34-47, Feb. 1994.

[4] M. Stricker, and M. Orengo, "Similarity of Color Images," in Proc. SPIE Storage and Retrieval for Image and Video Databases, pp. 381-392, Feb. 1995.

[5] Y. Chen and J. Z. Wang, "A Region-Based Fuzzy Feature Matching Approach to Content- Based Image Retrieval," in IEEE Trans. on PAMI, vol. 24, No.9, pp. 1252-1267, 2002.

[6] A. Natsev, R. Rastogi, and K. Shim, "WALRUS: A Similarity Retrieval Algorithm for Image Databases," in Proc. ACM SIGMOD Int. Conf. Management of Data, pp. 395-406, 1999.

[7] J. Li, J.Z. Wang, and G. Wiederhold, "IRM: Integrated Region Matching for Image Retrieval," in Proc. of the $8^{\text {th }}$ ACM Int. Conf. on Multimedia, pp. 147-156, Oct. 2000.

[8] P. Howarth and S. Ruger, "Robust texture features for still-image retrieval", IEE. Proceedings of Visual Image Signal Processing, Vol. 152, No. 6, December 2005.

[9] Cao LiHua, Liu Wei, and Li GuoHui, "Research and Implementation of an Image Retrieval Algorithm Based on Multiple Dominant Colors",Journal of Computer Research \& Development, Vol 36, No. 1,pp.96100,1999 .
[10] J. R. Smith, F. S. Chang, "Tools and Techniques for Color Image Retrieval", Symposium on Electronic Imaging: Science and Technology-Storage and Retrieval for Image and Video Database IV, pp.426-237, 1996.

[11] H. T. Shen, B. C. Ooi, K. L. Tan, Giving meanings to www images," Proceedings of ACM Multimedia, 2000, pp.39-48.

[12] FAN-HUI KONG, "Image Retrieval using Both color and texture features" proceedings of the $8^{\text {th }}$ international conference on Machine learning and Cubernetics,Baoding,12-15 July 2009.

[13]. JI-QUAN MA, "Content-Based Image Retrieval with HSV Color Space and Texture Features", proceedings of the 2009 International Conference on Web Information Systems and Mining.

[14]. P.S.Hiremath, Jagadeesh Pujari, "Content based image retrieval using Color, Texture and Shape features", proceedings of the $15^{\text {th }}$ International conference on Advanced Computing and communications.

[15]. Wang's dataset http://wang.ist.psu.edu/

[16] Smith J R, Chang S F. Tools and techniques for color image retrieval, in: IST/SPIE-Storage and Retrieval for Image and Video Databases IV, San Jose, CA, 2670, 1996,426-437

[17] Chia-Hung Wei, Yue Li, Wing-Yin Chau, Chang-Tsun $\mathrm{Li}$, Trademark image retrieval using synthetic features for describing global shape and interior structure, Pattern Recognition 42 (3) (2009) 386-394.

[18] Rui Y, Huang T S, Chang S F. Image retrieval: current techniques,promising directions and open issues, Journal of Visual Communication and Image Representation, 1999, 10( I): 39-62

[19] Song Mailing, Li Huan, "An Image Retrieval Technology Based on HSV Color Space", Computer Knowledge and Technology, No. 3,pp.200-201, 2007.

[20] B S Manjunath, W Y Ma, "Texture feature for browsing and retrieval of image data",IEEE Transaction on PAMI, Vol 18, No. 8, pp.837-842. 